\title{
Effectiveness of quadriceps muscle strengthening on knee joint stability and activities of daily living in patients with knee joint osteoarthritis
}

\author{
Kumarahewa $\mathrm{CV}^{1}$, Amaratunga $\mathrm{HA}^{2}$ \\ ${ }^{1}$ Physiotherapy Unit, Teaching Hospital Ragama \\ ${ }^{2}$ Department of Anatomy, Faculty of Medicine, University of Peradeniya, Peradeniya, \\ Sri Lanka
}

Running title: guided quadriceps exercises for knee osteoarthritis

\begin{abstract}
Objectives: Knee is the largest weight bearing joint in the body and thereby is affected by diseases such as osteoarthritis more frequently. The aim of this study was to evaluate the effect of a 4 week physiotherapy exercise program, designed to improve quadriceps muscle strength and girth, and to observe its effect on knee pain and activities of daily life through a pre and post exercise analysis in patients with knee joint osteoarthritis (OA).
\end{abstract}

Methods: Thirty patients diagnosed with knee joint osteoarthritis irrespective of gender, over the age of 45 and less than 75 years were recruited for the study. Initially knee joint pain severity was assessed using the VAS (visual analogue scales) and WOMAC questionnaire was used to assess the activities of daily living. The thigh circumference was measured in centimeters. Data was analyzed by SPSS 20.

Results: Mean age of osteoarthritis patients was 54 years. There was a statistically significant, reduction in knee pain ( $p=0.001)$, improvement of quadriceps muscles girth $(p=0.012)$ and an improvement of daily living activities $(p=0.001)$ after engaging in the physiotherapy exercise program.

Conclusion: A four week guided quadriceps muscle strengthening exercise program can significantly increase the quadriceps muscles girth and knee joint stability and reduce pain and improve the activities of daily living in patients with knee OA.

Keywords : Osteoarthritis, WOMAC, VAS

\section{Introduction}

The knee joint is one of the largest joints in the body and is a complex synovial joint of the hinge variety. It is a weight bearing joint and tends to get affected by diseases such as osteoarthritis (OA). OA is regarded as a degenerative joint disease characterized by reduction of the thickness of the articulate cartilage in a focal manner (1). It is diagnosed commonly in adults over the age of 40 years and it's reported that most people will have some features of OA in their weight- 
Kumarahewa CV, Amaratunga HA - Effectiveness of qudreceps muscle strengthening on knee joint stability and activities of daily living in patients with knee joint osteoarthritis

bearing joints at the age of 60 years.

The disease becomes symptomatic in around $15 \%$ of all adults over the age of 40 and its impact can be debilitating (2). The knee is the joint most commonly affected by this disease and is characterized by joint pain that increases with activity, night pain, morning stiffness, limited joint motion, reduced muscles girth, crepitus, soft tissue swellings, deformity and reduction of physical function (3).

Muscles such as the quadriceps, hamstrings and the illiotibial tract that surround the knee play an important role in protecting the joint by absorbing shock during weight bearing and movements of the knee. Strengthening exercises which improve and maintain muscle strength stabilizes and protects the joints, decreasing pain and stiffness and reducing loss of cartilage. Nonsurgical treatment of OA includes, medications, weight loss, muscle strengthening and bracing (4). Strengthening exercises, for the quadriceps muscle, has been reported to reduce pain and disability in knee $\mathrm{OA}$

Several studies have proven the effect of hospital based and home based exercise programs to increase muscle strength and have reported reduction in pain and stiffness and improvement in physical function $(6,7,8)$. With this background it was the aim of this study to assess the importance of a four week exercise program, designed to improve quadriceps muscles girth, on knee pain and activities of daily living in patients diagnosed with knee joint OA.

\section{Materials and methods}

Thirty patients over the age of 45 and less than 75 years, diagnosed with knee joint $\mathrm{OA}$ attending the physiotherapy Department at Teaching Hospital Ragama were recruited. Ethical clearance was granted by the ethical review committee of the Faculty of Medicine University of Peradeniya. Written informed consent was sort from all patients. Patients with a history of knee, hip or ankle injuries or surgery, those patients who claimed to have no knee pain and those who had received a cortisone injection to the knee joint within the previous 30 days were excluded.

The VAS a single dimension horizontal scale, which consists of a $10 \mathrm{~cm}$ line on which participants rate their pain from 0-10 established by Creamer, Lethbridge-cejku and Hochberg (1999) was used to measure the degree of knee joint pain. The WOMAC questionnaire designed according to the McMaster Universities osteoarthritis index was used to assess the activities of daily living (9). This is a widely used questionnaire which rates pain during different physical activities and the ability to perform day to day work. Each of the participants was scored with the VAS and the WOMAC questionnaire prior to implementing the exercise program.

Quadriceps muscle girth was measured as the thigh circumference. Measurement of thigh circumference was performed $15 \mathrm{~cm}$ proximal to the superior pole of the patella.

Measurement was taken while the 
Kumarahewa CV, Amaratunga HA - Effectiveness of qudreceps muscle strengthening on knee joint stability and activities of daily living in patients with knee joint osteoarthritis

patient was in the supine position. The thigh circumference was measured in centimeters with a measuring tape on both legs as described by Robert and Laprade (2012).

Next the exercise program was explained in detail to each patient at the first visit to the physiotherapy unit. During the consequent visits the treating therapist observed the exercise program at each session and guided and aided the patients in performing the exercises. This exercise program is a predesigned program conducted at the center for quadriceps strengthening routinely.

The exercise program consisted of a warm-up on a stationary bicycle or walking for 10 minutes. Next the patients were asked to do quadriceps, hamstring, and iliotribial band, stretches to hold for 20 seconds and repeat 3 times. Thereafter, Isometric vastus medialis contraction in full extension held for five seconds, Quadriceps contraction in full extension held for five seconds, Straight leg raising exercise holding five seconds, Isokinetic quadriceps contraction full extension for five seconds, Isokinetic hamstring contraction, Isokinetic hip extension in prone position and Isokinetic hip abduction inside line position was done with 30 to 45 seconds interval between exercises. Exercises were progressed by increasing weight according to $10 \mathrm{RM}$ by using sand bags. The participants performed these exercises twice a week at the physiotherapy unit under the guidance of the therapist. Exercises were practiced in a painless or minimally painful manner. Patients were followed up for 4 weeks.

At the end of the 4 week exercise program the patients were reassessed using the VAS pain scale, WOMAC questionnaire and measurement of muscles girth.

Data was analyzed using Statistical Package for Social Sciences (SPSS) 20. The pre and post exercise program scores were compared separately for pain, WOMAC score and muscle girth. The pre and post exercise program values of the VAS and the WOMAC score (activities of daily life score) were analyzed using Wilcoxon signed ranks test for related samples. Quadriceps girth was analyzed using the paired sample $t$ test to evaluate the changes between the values obtained before and after the exercise program.

Mean, standard deviations were calculated for pain, muscles girth, stiffness, and physical function. Nonparametric Wilcoxon test used because of limited sample size and nonnormalized distribution assessed pre and post intervention. An alpha level of 0.05 and a p-value $<0.05$ was taken as significant.

\section{Results}

Out of thirty OA patients $90 \%(\mathrm{n}=27)$ were females. The mean age of the patients was 54 years. Duration of the symptoms varied from 1 month to 120 months. 
Kumarahewa CV, Amaratunga HA - Effectiveness of qudreceps muscle strengthening on knee joint stability and activities of daily living in patients with knee joint osteoarthritis

All of the patients showed statistically significant reduction in their knee pain assessed by VAS, knee pain during activities of daily living and joint stiffness after going through the exercise program. Mean values of pre and post-test with standard deviations is given in Table 1. Out of the 30 patients only 27 patients complained of stiffness and all 27 showed reduction in their knee joint stiffness after going through the exercise program. Mean percentage reduction of stiffness was $30.1 \%$.

Wilcoxon test revealed statistically significantly reduction in joint stiffness after the exercise program (Table 1). value was $47.72, \mathrm{SD} \pm 4.974$. This was analyzed by paired $t$ test and $p$ value of 0.012 was observed. There is a statistically significant increase in the quadriceps muscle girth after going through the exercise program $(p<0.05)$.

\section{Discussion}

The knee joint is essential for activities of daily living. Running walking sitting and squatting put great strain on the knee joint. Knee joint pain during activities of daily living is the commonest reason for patients with OA to seek medical interventions. The knee

Table1: pre and posttest values for VAS score, activities of daily living, joint stiffness and physical function with the comparisons

\begin{tabular}{|l|l|l|}
\hline \multicolumn{1}{|c|}{ Variable } & \multicolumn{1}{|c|}{ Mean(SD) } & \multicolumn{1}{|c|}{ p value } \\
\hline VAS pre-test pain & $6.77( \pm 1.073)$ & \multirow{2}{*}{0.001} \\
\hline VAS posttest pain & $4.37( \pm 1.866)$ & \\
\hline Pre-test pain during daily activities & $5.90( \pm 3.408)$ & \multirow{2}{*}{0.001} \\
\cline { 1 - 2 } Post-test pain during daily activities & $3.60( \pm 2.895)$ & \multirow{2}{*}{0.001} \\
\cline { 1 - 2 } Pre- test joint siffness & $1.78( \pm 1.316)$ & \\
\cline { 1 - 2 } Post-test jointstiffness & $1.06( \pm 1.048)$ & \multirow{2}{*}{0.001} \\
\cline { 1 - 2 } Pre-test physical function & $25.90( \pm 11.158)$ & \\
\cline { 1 - 2 } Post-test physical function & $18.00( \pm 9.52)$ & \\
\hline
\end{tabular}

Table 2 shows that the exercise program resulted in significant changes in quadriceps muscle girth. Pre exercise program quadriceps muscle girth mean was 47.61, $\mathrm{SD} \pm 4.996$. Post exercise program quadriceps muscle girth mean joint is stabilized by the muscles and ligaments that surround it.

Strengthening exercises improve muscle bulk thereby reducing joint pain and improving activities of daily living. Muscle strength is defined as the ability

Table 2: Comparison of pre and post test means of quadriceps muscle girth

\begin{tabular}{|l|l|l|l|l|l|l|l|}
\hline Characteristic & \multicolumn{2}{l|}{$\begin{array}{l}\text { Pre } \\
(\mathrm{n}=30)\end{array}$} & \multicolumn{2}{l|}{$\begin{array}{l}\text { Post } \\
(\mathrm{n}=30)\end{array}$} & T(df) & $\mathrm{P}$ & $\begin{array}{l}95 \% \\
\text { ci interval }\end{array}$ \\
\hline & Mean & SD & Mean & SD & & & \\
\hline $\begin{array}{l}\text { Quadriceps } \\
\text { muscles girth }\end{array}$ & 47.61 & 4.996 & 47.72 & 4.974 & $\begin{array}{l}-2.693 \\
(29)\end{array}$ & 0.012 & $\begin{array}{l}-0.194- \\
-0.027\end{array}$ \\
\hline
\end{tabular}


Kumarahewa CV, Amaratunga HA - Effectiveness of qudreceps muscle strengthening on knee joint stability and activities of daily living in patients with knee joint osteoarthritis

of a muscle to produce force (10) and increases with training. Our study used strengthening exercises based on 10 repetitions maximum (10RM) with progressively increasing weights using sand bags and the quadriceps bench to improve the quadriceps muscle strength.

Our study reports a statistically significant reduction of knee joint pain with improvement of activities of daily living, after engaging in an intense guided exercise program for 4 weeks. The study done by Baker (2001) (11) illustrated that high intensity home based strengthening training significantly improves muscle strength with reduction of joint pain, and improvement of physical function and quality of life of OA patients. They also report that self-reported pain improved by $36 \%$ and physical function by $38.7 \%$.

The study done by Fisher (2015) (12) and Van baar et al. (1998) (13) reports the effectiveness of exercise therapy in patients with OA of the hip or knee in reduction of pain and disability. The present study also found that the exercise program significantly improved the activities of daily living.

In a study conducted by Deyle (2005) (14) the treatment group attended 8 treatment session of a physiotherapy exercise program and the WOMAC improved by $52 \%$ (535mm, $95 \%$ $\mathrm{CI}=426-644)$. Jorge (2015) (15) conducted a study on women with osteoarthritis of the knee and reports that progressive resistance exercise has a significant effect in reducing pain and improving function, some quality of life and strengthen the female knees by strengthening the muscles surrounding the knee. Similar findings were observed in our study with a significant reduction in knee joint stiffness, improvement of day to day activities and reduction of pain.

Further a study conducted by Thomas, et al. (2002) (16) reports a highly significant reduction in pain in a group of knee OA patients who underwent an exercise program for 6 weeks with that of a group who did not engage in exercise. They further report that direct contact with physical therapist has a much better improvement rate. In the current study the physiotherapist was always in contact with the patients guiding and aiding them in all aspects of the exercises and the significant improvement in muscle girth and reduction in pain was observed even though this study was done for only 4 weeks.

In our study a statistically significant reduction in joint stiffness was observed after the exercise program $(\mathrm{p}<0.05)$ of only 4 week, while Shakoor, et al. (2007) (17) reports similar findings in a longer duration program.

Cooper (1981) (18) states that increasing muscles girth reflects increasing muscles power and strength. Therefore it is rational to measure the thigh circumference as a measure of quadriceps muscle strength and our findings of the increase of the thigh 
Kumarahewa CV, Amaratunga HA - Effectiveness of qudreceps muscle strengthening on knee joint stability and activities of daily living in patients with knee joint osteoarthritis

circumference indirectly prove the increase in quadriceps power. A statistically significant improvement in quadriceps muscles girth was observed in our group $(\mathrm{p}<0.05)$.

This to our knowledge is the first study in Sri Lanka to assess the effects of a physiotherapy exercise program on quadriceps muscles girth, knee pain and activities of daily living in knee OA patients. Our findings suggest that a 4 week intensive physiotherapy program with high therapist contact is extremely useful in rehabilitation of patients with $\mathrm{OA}$ of the knee.

\section{Conflict of interest}

None declared.

\section{Correspondence -}

Dr. (Mrs.) H.A. Amaratunga,

Department of Anatomy,

Faculty of Medicine,

University of Peradeniya,

Peradeniya, 20400,

Sri Lanka.

E-mail: himanipeiris@yahoo.com, himanip@pdn.ac.lk

Tel: +94 812396275

Mobile +94 773920177

Fax: +94 812389106

\section{References}

1. Di Cesare, P, Abramson S, Samuels J. Kelley's Textbook of Rheumatology. $8^{\text {th }}$ ed. Philadelphia, Pa.: Saunders Elsevier 2009; 15251540.
2. Felson D. An update on the pathogenesis and epidemiology of osteoarthritis. Radial Clin North Am 2004;42:1-9

3. Thomson A, Skinner A, Piercy 1991 J. Tidy'physiotherapy, $12^{\text {th }}$ edn, Butterworth Heinemann, Oxford, 108.

4. Mushtaq S, Choudhary R, Scanzello, CR. Non-surgical treatment of osteoarthritis - related pain in the elderly. Currnt Reviews in Muscluloskeletal Medicine 2011;4 (3): 113-122

5. Jamtvedt G, Dahm KT, Holm I, Flottorp S. Measuring physiotherapy performance in patients with osteoarthritis of the knee. BMC health services research 2008; 8 (1): 145.

6. Thomas KS, Muir KR, Doherty M, Jones AC, O'Reilly SC, Bassey EJ. Home based exercise programme for knee pain and knee osteoarthritis. British medical Journal 2002;325:752

7. Deyle GD, Henderson N E, Matekel RL, Ryder MG, Garber MB, Allison SC. Effectiveness of manual physical therapy and exercise in osteoarthritis of the knee. Annals of internal medicine 2000;132(3):173-181.

8. McCarthy CJ, Mills PM, Pullen R, Richardson G, Hawkins N, Robert CR, Silman AJ, Oldham JA. Supplementation of a home-based exercise programme with a class- 
Kumarahewa CV, Amaratunga HA - Effectiveness of qudreceps muscle strengthening on knee joint stability and activities of daily living in patients with knee joint osteoarthritis

based programme for people with osteoarthritis of the knees. Health Technol Assess 2004;8 (46):1-61.

9. McConnell S, Kolopack P, Davis AM. The Western Ontario and McMaster Universities Osteoarthritis Index (WOMAC). Arthritis care and research 2001;45 (5):453-461.

10. Sayers SP. High-speed power training: a novel approach to resistance training in older men and women. J Strength Cond Res 2007;21:518-526.

11. Baker KR, Nelson ME, Felson DT, Layne JE, Sarno R, Roubenoff R. The efficacy of home based progressive strength training in older adults with knee osteoarthritis. The journal of Rheumatology 2008;(7):1655-1665

12. Fisher NM, Prendergast DR, Gresham GE, Calkins E. Muscle rehabilitation: its effect on muscular and functional performance of patients with knee osteoarthritis. Arch Phys Med Rehabil 1991;72:367-374

13. Van Baar ME, dekker J, Ootendrop RA, Bijl TB, Lemmens JA,
Bijlsma JW. The effectiveness of exercise therapy in patient with osteoartheritis of the hip or knee. J Rheumatol 1998;25(12):2432-9.

14. Deyle Gail D, et al. Physical therapy treatment effectiveness for osteoarthritis of the knee. Physical therapy 2005;85:(12), 1301.

15. Jorge RTB. Progressive resistance exercise in women with osteoarthritis of the knee. Clinical rehabilitation 2014;2(239):10 - 64

16. Thomas, DeLorm, Todd JS, Shurley JP, Todd TC.Thomas L. DeLorme and the science of progressive resistance exercise. J Strength Cond Res 2012;26 (11): 2913-23

17. Shakoor N, Furmanov S, Nelson DE, Block JA. Pain and its relationship with muscle strength and proprioception in knee OA. $\mathrm{j}$ musculoskeltal neuronal interact 2007;8 (1):35-42

18. Cooper H, Dodds WN, Adams FT, Dedombal FT, Steele VA. Use and misuse of the tape-measure as a means of assessing muscle strength and power Rheumatol Rehabil 1981;20(4):211-8. 